\title{
PROSTATE ANTIGEN LEVELS IN LOCALLY ADVANCED PROSTATE CANCER PATIENTS TREATED WITH HIGH ENERGY NEUTRONS AT FERMILAB
}

\author{
K. R. Saroja, M.D. ${ }^{1}$, L. Cohen, M.D. ${ }^{2}$, F. R. Hendrickson, M.D. ${ }^{1}$, JoAnne Mansell, R.N.,P.A.,O.C.N. ${ }^{3}$ \\ ${ }^{1}$ Rush Presbyterian St. Luke's Hospital, Radiation Therapy Department, Chicago, II 60612 \\ ${ }^{2}$ Michael Reese Hospital/University of Chicago, Radiation Therapy Department, Chicago, II 60637 \\ ${ }^{3}$ Fermi National Accelerator Laboratory, Neutron Therapy Facility, Batavia, II 60510
}

\begin{abstract}
The prostate specific antigen values were analyzed retrospectively for prognostic implications in thirty-nine locally advanced (Stages $C$ and D1) prostate cancer patients treated using high energy neutrons at the Midwest Institute for Neutron Therapy at Fermilab. These patients were treated between January 1981 and March 1989. Four groups of patients were identified. Group I includes patients whose PSA values reached normal $(0-4 \mathrm{ng} / \mathrm{ml})$ following neutron therapy and remained so subsequently. Of the fourteen patients in this group, twelve $(86 \%)$ were disease free, with follow-up periods of 18 to 50 months. Only two of these fourteen patients were diagnosed with distant metastatic disease. A rapid fall of prostate specific antigen levels, with mean half life of $45( \pm 10)$ days, was observed in these patients.
\end{abstract}

There were thirteen patients in Group II with PSA levels which remained elevated. Distant failure alone occurred in eight $(62 \%)$ and as a component in two (15\%) additional patients. Two of thirteen patients (15\%) has local persistence of tumor. Only one patient in this group was free of disease at 1 year post treatment. The mean half life of PSA in this group was $131( \pm 24)$ days.

Group III consisted of nine patients with only an initial drop in the antigen levels. Of the nine, four $(44 \%)$ were diagnosed with distant metastases and one of these failed locally as well. Three patients had local persistence of tumor. Two other patients had no clinical evidence of overt disease. Half life of the antigen was $94( \pm 27)$ days.

There were three patients in Group IV whose PSA levels slowly decreased although a normal baseline has not been achieved. They are all clinically well.

Our preliminary observation is that the rapid normalization of PSA following neutron irradiation can be used to identify prostate cancer patients with good prognosis. However this observation needs confirmation in a larger series of patients followed for a longer period of time. Whether the early therapeutic intervention of hormonal manipulation for patients in Groups II, III, and IV is warranted and would alter their long term prognosis needs to be investigated.

\section{Introduction}

Prostate specific antigen (PSA) is a glycoprotein produced exclusively by prostate tissue. This glycoprotein was first isolated in 1979 by Wang and Associates at the Rosewell Park Memorial Institute. The clinical conditions where the antigen is elevated are benign prostatic hypertrophy, prostatitis, local and metastatic prostate cancer. Only recently has the serum concentration of prostate specific antigen become generally recognized as an important tumor marker for an early detection and monitoring of prostate cancer. In patients with prostate cancer, the serum concentration of PSA is found to be proportional to the tumor volume and disease stage. ${ }^{1,2}$ The antigen is determined by using sensitive immunoassay and reaches to undetectable levels following successful radical prostectomy. ${ }^{1}$ Megavoltage photon radiation and/or $I^{125}$ radioactive seed 
implantation are also generally accepted methods to treat locally confined prostate cancer. PSA determination is used as an indicator of response to irradiation. 3,4

High energy fast neutrons are used to treat patients with locally advanced prostate cancer at the Neutron Therapy Facility at Fermilab. We are reporting the results of a retrospective analysis of PSA subsequent to neutron beam therapy in these patients.

\section{Materials and Methods}

The prostate specific antigen values were analyzed retrospectively for prognostic implications in thirty-nine prostate cancer patients treated using high energy neutrons. These patients were treated between Januay 1981 and March 1989. There were 25 patients with Stage C and 11 patients with Stage D1 disease. Three patients had clinical large B2 or C disease. Prostate specific antigen determinations were performed using previously frozen sera, by immunoradiometric assay. (Tandem-R PSA assay, Hybritech Inc.). The normal range is $0-4.0 \mathrm{ng} / \mathrm{ml}$.

All the patients received external neutron beam therapy. The whole pelvis was treated to a dose of $14 \mathrm{~Gy}$, delivered in 8 fractions. The prostate received an additional $7 \mathrm{~Gy}$ in 4 fractions as a boost to carry the total dose to $21 \mathrm{~Gy}$. The overall treatment period was approximately four weeks, giving 3 fractions per week to a total of 12 fractions. PSA was determined 3-4 months following completion of therapy and once every three months until the normal baseline is reached. From then on PSA was repeated once every 6 months. Appropriate investigations (CT scans of the pelvis, transrectal ultrasound followed by with or without biopsy, and bone scan) were done as clinically warranted.

The prostate specific antigen values were normalized assuming the initial reading to be $100 \%$. Subsequent readings taken at various intervals following treatment were expressed as a percentage of the initial value. Half life was determined for each subject by a linear regression of logarithm of the normalized value as a function of elapsed time from completion of treatment. From two to four readings were sufficient to determine the downward slope of the line as shown in the graph. The mean half life ranged from 5 to 250 days for the entire group.

Disease Status of Patients in Various P.S.A. Groups

\begin{tabular}{|c|c|c|c|c|c|c|c|}
\hline Group & $\begin{array}{c}\text { No. of } \\
\text { Pts. }\end{array}$ & $\begin{array}{c}\text { Disease } \\
\text { Free }\end{array}$ & $\begin{array}{c}\text { Local } \\
\text { Control }\end{array}$ & $\begin{array}{c}\text { Local } \\
\text { Persistence }\end{array}$ & $\begin{array}{c}\text { Local } \\
\text { Rec. Only }\end{array}$ & $\begin{array}{c}\text { Local } \\
+ \text { D.M. }\end{array}$ & $\begin{array}{c}\text { D.M. } \\
\text { Only }\end{array}$ \\
\hline I & 14 & $\begin{array}{c}12 \\
(86 \%)\end{array}$ & $\begin{array}{c}13 \\
(93 \%)\end{array}$ & - & - & $\begin{array}{c}1 \\
(7 \%)\end{array}$ & $\begin{array}{c}1 \\
(7 \%)\end{array}$ \\
\hline II & 13 & $\begin{array}{c}1 \\
(8 \%)\end{array}$ & $\begin{array}{c}9 \\
(69 \%)\end{array}$ & $\begin{array}{c}2 \\
(15 \%)\end{array}$ & - & $\begin{array}{c}2 \\
(15 \%)\end{array}$ & $\begin{array}{c}8 \\
(62 \%)\end{array}$ \\
\hline III & 9 & $\begin{array}{c}2 \\
(22 \%)\end{array}$ & $\begin{array}{c}5 \\
(56 \%)\end{array}$ & $\begin{array}{c}1 \\
(11 \%)\end{array}$ & $\begin{array}{c}2 \\
(22 \%)\end{array}$ & $\begin{array}{c}1 \\
(11 \%)\end{array}$ & $\begin{array}{c}3 \\
(33 \%)\end{array}$ \\
\hline Total & 36 & $42 \%$ & $75 \%$ & $8.3 \%$ & $5.6 \%$ & $11 \%$ & $33 \%$ \\
\hline
\end{tabular}

Group I: Normal PSA post neutron therapy.

Group II: Persistently elevated PSA.

Group III: Initial decrease to normal values then subsequent increase in PSA. 


\section{Results}

Four groups of patients were identified depending on their post therapy PSA levels.

Group I: Patients in whom PSA reached normal $(4 \mathrm{ng} / \mathrm{ml})$ and remained so. The average half life of the antigen was 51 days. There were 14 patients in this group. Twelve of these fourteen patients $(86 \%)$ are alive and disease free. Thirteen patients have local control (93\%). Follow-up period is 18 to 50 months.

Group II: In this group of patients PSA remained elevated. The average half life of the antigen was 131 days. Of thirteen patients in this group, only one has no evidence of disease (8\%) 14 month post therapy. The tumor is locally controlled in nine of thirteen (69\%) patients. Follow-up period is 12 to 72 months.

Group III: Nine patients belonged to this group. There was an initial drop of their PSA level to normal and then a subsequent increase. The mean half life of the antigen was 94 days. Two patients in this group are disease free $(22 \%)$. Follow-up period is 12 to 60 months. Overall local tumor control rate for this group is $56 \%(5 / 9)$.

Group IV: There are three patients in this group. There has been a gradual decrease in PSA although normal baseline has not been achieved. The follow-up period for this group is 7 to 13 months. The definite evidence of either presence or absence of prostate cancer in these patients was not clear at the time of this analysis. Longer period of follow-up would probably help to categorize these patients.

A total of 36 patients from Groups I, II, and III were analyzed for their disease status and the details of the results are shown in the Table. Local tumor control, rate of metastases, and disease free status of these patients is depicted in histograms.

\section{Conclusions}

Our retrospective analysis of PSA levels in prostate cancer patients treated with neutron beam therapy has led us to draw these preliminary conclusions:

1. PSA is a very sensitive tumor marker for the diagnosis of early, recurrent and/or metastatic prostate cancer. It can be used as an indicator of tumor response to neutron beam therapy.

2. PSA has a predictive prognostic implication. The patients in whom PSA levels decrease rapidly to normal, and remains so, have a good prognosis. Serial determinations of post treatment PSA helps to identify these patients. (Group I: Local control rate of $93 \%$ and disease free rate of $86 \%$ ).

The patients in whom the PSA remains elevated or increases after initial drop to normal have poor prognosis. (Group II \& III: Local control rate of $64 \%$ and disease free rate of $14 \%$ ). Repeat diagnostic work-up for the presence of local and/or metastatic disease may be indicated before the overt manifestation of disease.

3. The patients in whom PSA drops slowly (Group IV) need a longer period of follow-up.

Whether early therapeutic intervention for patients in Groups II \& III is warranted, and would alter their prognosis certainly needs to be determined from the ongoing and future studies. 


\section{References}

1. Oesterling, J.E., Chan, D.W., Epstein, J.I., Kimball, A.W., Brwzek, D.J., Rock, R.C., Brendler, C.B., Walsh, P.C. Prostate Specific Antigen in the Preoperative and Postoperative Evaluation of Localized Prostatic Cancer Treated with Radical Prostatectomy. J. Urol. 139: 766-772, 1988.

2. Stamey, T.A., Yang, N., Hay, A.R., McNeal, J.E., Freiha, F.S., Redwine, E. Prostate Specific Antigen as a Serum Marker for Adenocarcinoma of the Prostate. N. Engl. J. Med. 317: 909-916, 1987.

3. Landmann, C., Hunig, R. Prostatic Specific Antigen as an Indicator of Response to Radiotherapy in Prostate Cancer. Int. J. Radiation Oncology Biol. Phys., Vol. 17, pp. 10731076, 1989.

4. Dundas, S.G., Porter, T.A., Venner, M.P., prostate-specific Antigen. Monitoring the Response of Carcinoma of the Prostate to Radiotherapy with a New Tumor marker. Cancer 66:45481990 


\section{Local Control}

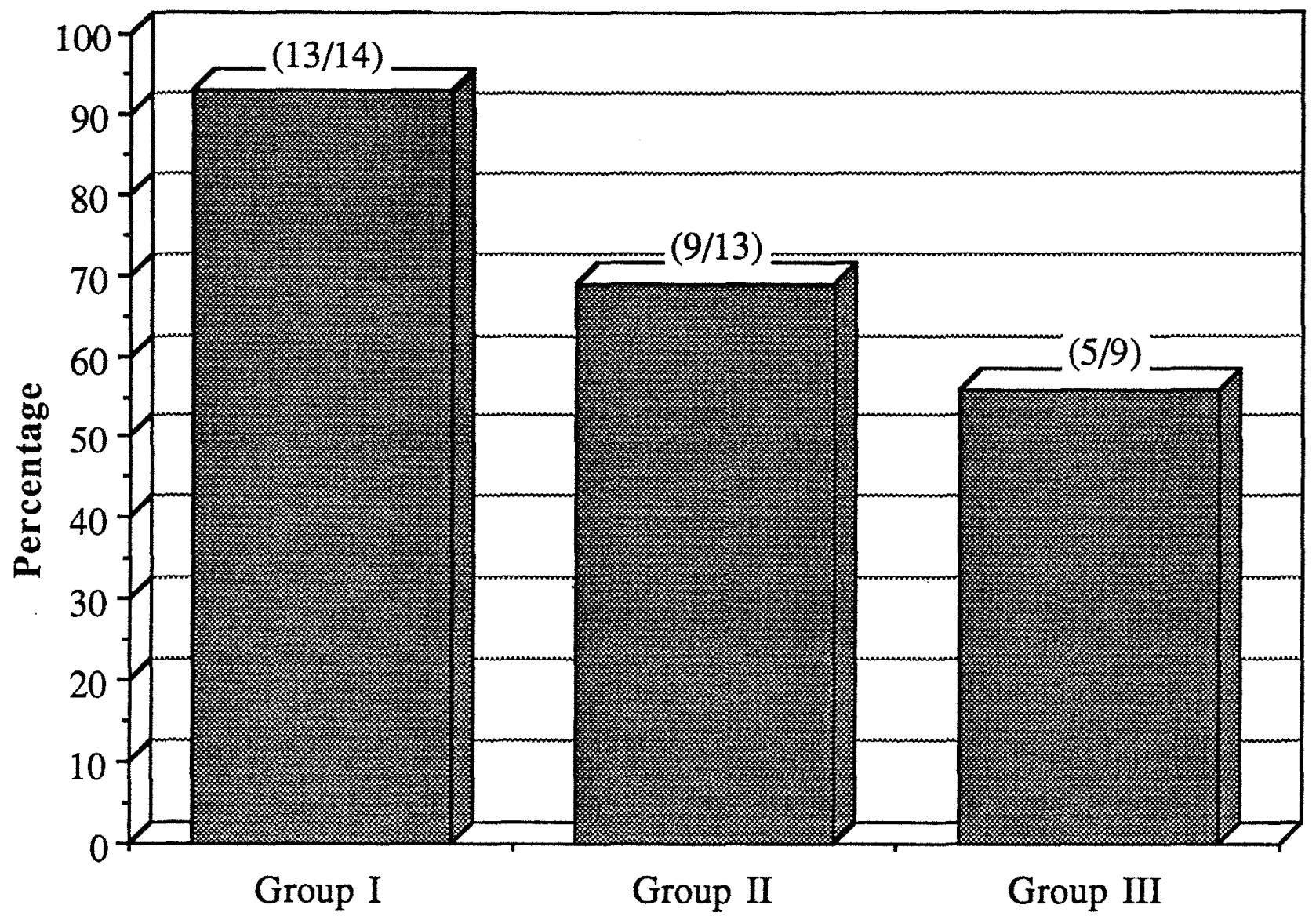

Group I: Normal PSA post neutron therapy.

Group II: Persistently elevated PSA.

Group III: Initial decrease to normal values then subsequent increase in PSA. 


\section{Distant Metastases}
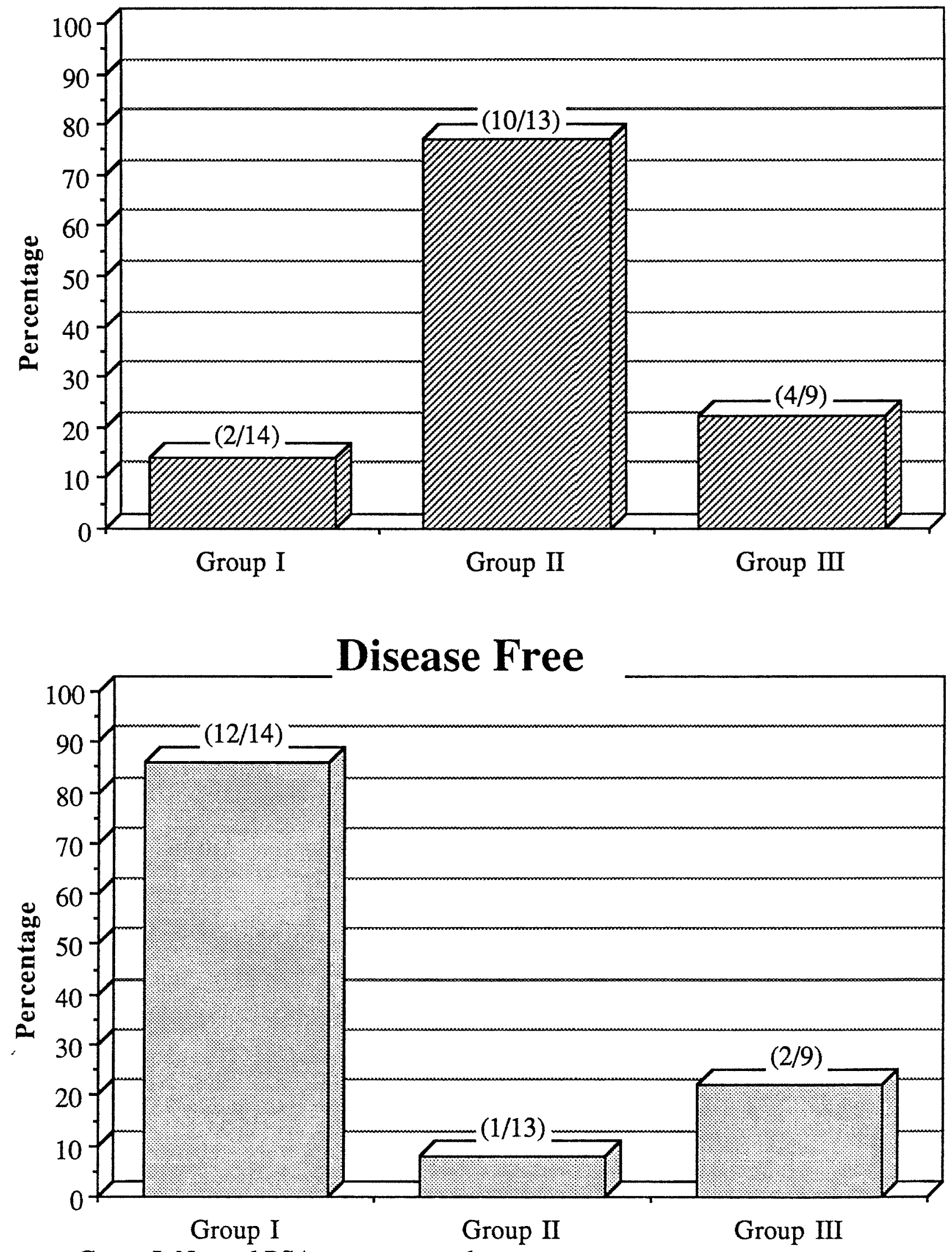

Group I: Normal PSA post neutron therapy

Group II: Persistantly elevated PSA.

Group III: Initial decrease to normal values then subsequent increase in PSA. 


\section{GROUP I REGRESSION LINES}

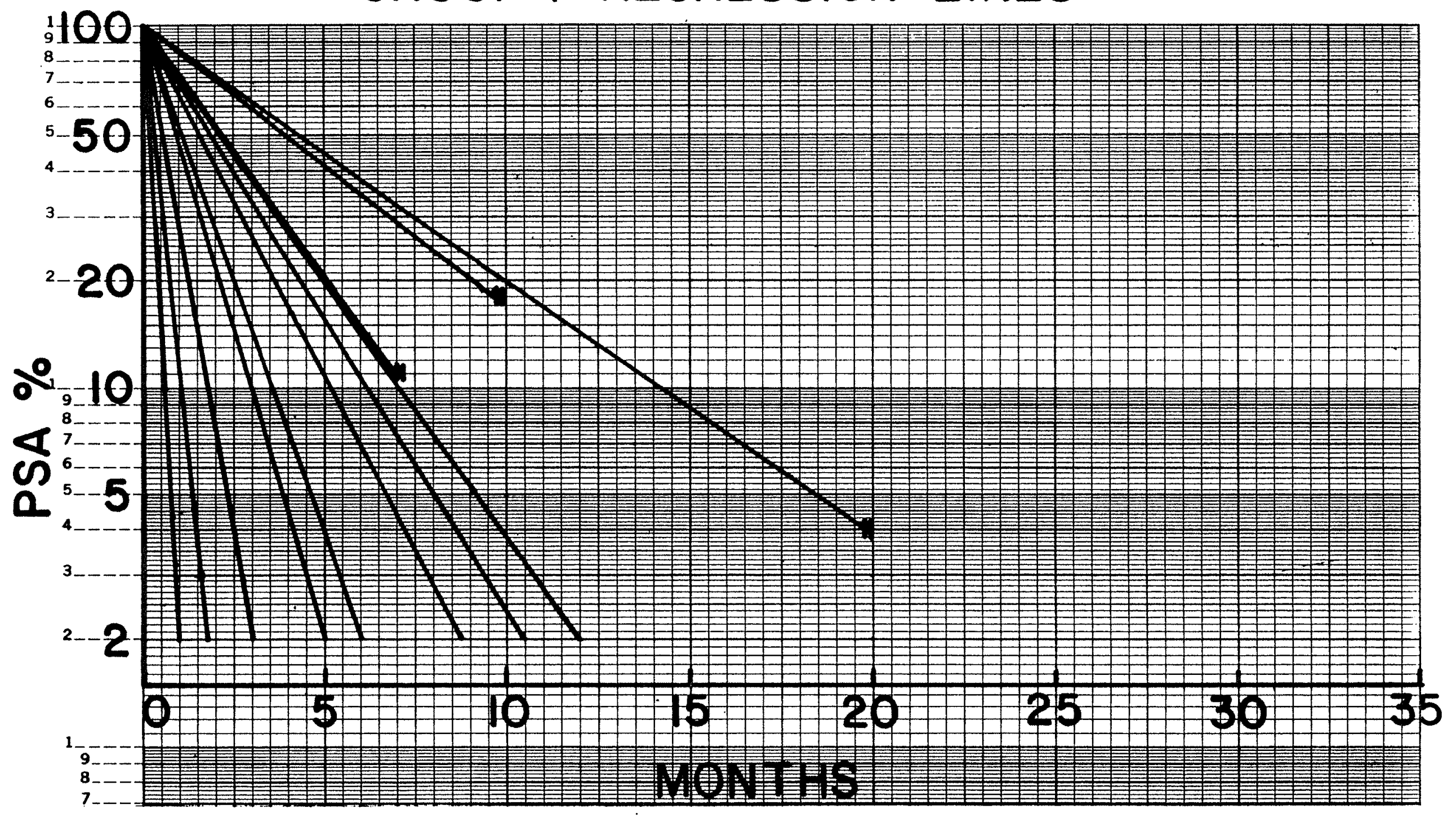




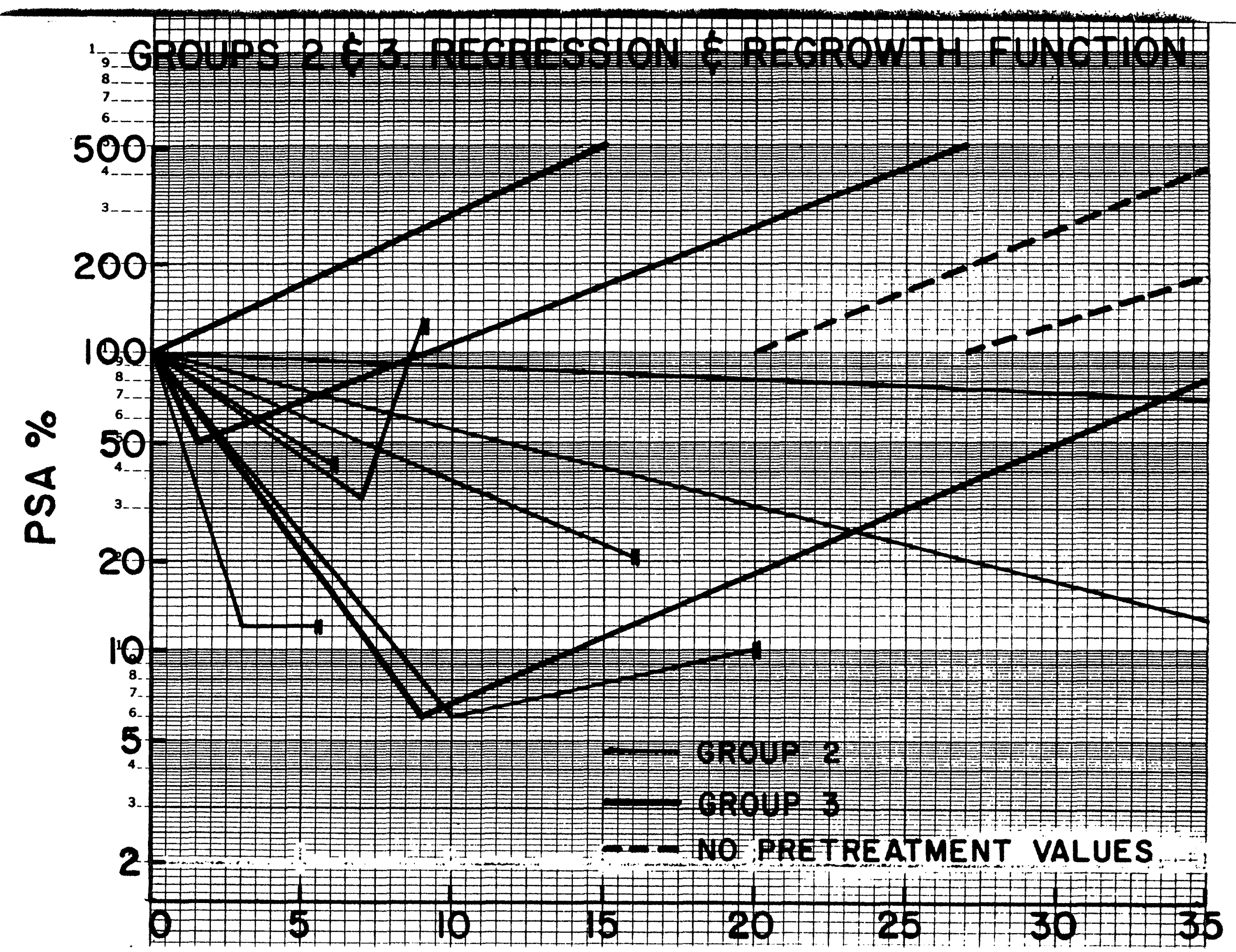

